\title{
Linking emotional distress to unhealthy sleep duration: analysis of the 2009 National Health Interview Survey
}

This article was published in the following Dove Press journal:

Neuropsychiatric Disease and Treatment

25 September 2015

Number of times this article has been viewed

\author{
Azizi A Seixas ${ }^{1}$ \\ Joao $\vee$ Nunes $^{2}$ \\ Collins O Airhihenbuwa ${ }^{3}$ \\ Natasha J Williams' \\ Seithikurippu Ratnas Pandi- \\ Perumal' \\ Caryl C James ${ }^{4}$ \\ Girardin Jean-Louis' \\ 'Center for Healthful Behavior \\ Change, Department of Population \\ Health, Division of Population Health, \\ New York University School of \\ Medicine, ${ }^{2}$ Sophie Davis School of \\ Biomedical Education, City College \\ of New York, New York, NY, USA; \\ ${ }^{3}$ Department of Biobehavioral Health, \\ The Pennsylvania State University, \\ State College, PA, USA; ${ }^{4}$ Department \\ of Sociology, Psychology and Social \\ Work, The University of the West \\ Indies, Mona, Jamaica
}

Objective: The objective of the study was to examine the independent association of emotional distress with unhealthy sleep duration (defined as $<7$ or $>8$ hours).

Methods: Data from the 2009 National Health Interview Survey (NHIS), a cross-sectional household survey, were analyzed to investigate the associations of emotional distress with unhealthy sleep durations, adjusting for sociodemographic factors, health risks, and chronic diseases through hierarchical multiple logistic regression analysis.

Participants: A total of 27,731 participants (age range 18-85 years) from the NHIS 2009 dataset were interviewed.

Measures: Unhealthy sleep duration is defined as sleep duration $<7$ or $>8$ hours, whereas healthy sleep is defined as sleep duration lasting for 7-8 hours. Emotional distress is based on the Kessler 6 Non-Specific Distress Battery, which assesses the frequency of feeling sad, nervous, restless, hopeless, worthless, and burdened, over a 30-day period.

Results: Of the sample, $51.7 \%$ were female; $83.1 \%$ were white and $16.9 \%$ were black. Eleven percent experienced emotional distress and 37.6\% reported unhealthy sleep. Adjusted logistic regression analysis revealed that individuals with emotional distress had $55 \%$ greater odds of reporting unhealthy sleep (odds ratio $[\mathrm{OR}]=1.55,95 \%$ confidence interval $[\mathrm{CI}]=1.42,1.68, P<0.001$ ).

Conclusion: Emotional distress, an important proxy for poor psychological health, was a significant predictor of unhealthy sleep, independent of the influences of several factors including demographic (age, education, sex, race/ethnicity, and family income), health risks (alcohol consumption and smoking status), and chronic diseases/conditions (diabetes, obesity, hypertension, heart disease, cancer, and arthritis).

Keywords: emotional distress, unhealthy sleep, sleep duration, sleep, mental health, depression, anxiety

\section{Introduction}

Mounting evidence indicates that unhealthy sleep (defined as short [ $<7$ hours] or long [ $>8$ hours] sleep durations) has a U-shaped relationship with chronic diseases. ${ }^{1-3}$ Specifically, unhealthy sleep increases individuals' risk for cardiovascular disease, cerebrovascular disease, diabetes, and mental illness. ${ }^{1-3}$ Unfortunately, despite efforts to consider sleep as an antecedent to chronic diseases, very little has been achieved regarding reduction of sleep-related chronic disease morbidity. Worse yet, to our knowledge, linkages between emotional distress and unhealthy sleep durations have not been systematically established. ${ }^{1,4}$

Over the last 3 decades, research has shown that sociodemographic, ${ }^{5,6}$ anthropometric, ${ }^{7,8}$ health risk, ${ }^{9}$ psychosocial, ${ }^{10}$ and genetic factors ${ }^{11}$ are all linked to sleep disorders ${ }^{12,13}$ as well as to specific aspects of sleep such as sleep architecture ${ }^{14}$ and sleep 
duration. ${ }^{3,5,15}$ Understanding the relationship between psychological/emotional factors and sleep duration is particularly important, as it presents the greatest opportunity to design interventions to effectuate behavioral changes. ${ }^{16}$ Evidence indicates that emotional distress is associated with changes in sleep architecture, total sleep time, sleep quality, sleep efficiency, rapid eye movement sleep, sleep onset latency, and slow wave sleep. ${ }^{10,17,18}$ Moreover, both short and long sleep durations are associated with mental health outcomes ${ }^{19}$ such as depression, ${ }^{20}$ anxiety, ${ }^{21}$ and suicidal behavior, ${ }^{22}$ it is believed that these mental health-related factors might explain the relationship between unhealthy sleep durations and chronic diseases. ${ }^{1}$ The purpose of our study was to investigate the association between emotional distress and unhealthy sleep duration ( $<7$ or $>8$ hours), while assessing the relative contribution of other known medical risk factors using the National Health Interview Survey (NHIS).

\section{Methods}

\section{Participants}

The NHIS (Adult File) is a nationally representative epidemiological study of noninstitutionalized adults in the USA that assesses a variety of health conditions and outcomes. In the NHIS 2009 dataset, 27,731 participants (age range 18-85 years) were interviewed face-to-face. To achieve survey objectives, we adjusted for utilization of clustering, stratifying, and oversampling methods for specific population subgroups such as non-Hispanic blacks and individuals aged 65 years and older.

\section{Procedures}

Data were collected from noninstitutionalized civilians from the 50 US states and the District of Columbia using a multistage area probability design sampling, with a conditional response rate of $65.4 \%$. Sociodemographic and physiciandiagnosed chronic disease data were obtained through face-toface interviews using computer-assisted personal interviewing (CAPI). CAPI is a computer program that guides interviewers during questionnaire administration and identifies, through an algorithm, inconsistencies and/or patterns between responses and other data collected during the interview process. For our study, we merged Adult, Person, Family, and Household files at the individual level to investigate the association between emotional distress and unhealthy sleep duration.

\section{Measures}

\section{Emotional distress}

Emotional distress, Kessler 6 Mental Health Scale (K6), is a six-item scale created for the NHIS to assess general psychological distress. ${ }^{23}$ On a five-point Likert scale $(0=$ "None of the time" to $4=$ "All of the time"), respondents were asked how often they experienced symptoms of depression (eg, sadness, hopelessness, everything takes an effort, or worthlessness) or anxiety (nervous, restless, or fidgety) in the past 30 days. Total scores ranged from 0 to 24 , with lower scores representing low levels of emotional distress, and higher scores representing higher levels of emotional distress. Scores greater than 13 indicate the possibility of serious mental illness, which the NHIS defines as the presence of a Diagnostic and Statistical Manual (DSM) disorder not caused by substance abuse disorder. ${ }^{24}$ It is likely that there are participants with a mental disorder who fell outside of this cut-point - false negatives. Although we did not address potential false negatives in our main analysis, evidence shows that the scale has good reliability and ecological validity. ${ }^{24}$ It has a high internal consistency (Cronbach's alpha $=0.89$ ), good discrimination (receiver operating characteristic $[\mathrm{ROC}]=0.86)$, low sensitivity $(0.36)$, high specificity (0.96), and high overall accuracy of 0.92 . In our study, emotional distress was dichotomized, such that scores equal or greater than 13 were coded as " 1 " denoting the presence of emotional distress and scores lower than 13 were coded as " 0 " denoting the absence of emotional distress.

\section{Unhealthy sleep}

Unhealthy sleep (sleep duration $<7$ or $>8$ hours) was measured based on the responses of participants to the following question "On average, how many hours of sleep do you get in a 24-hour period?" Participants were instructed to provide responses using 1-hour increments. For example, a reported 6-hour and 20-minute sleep duration was rounded to 6 hours. Participants who reported sleeping 7-8 hours were considered healthy sleepers, while those who reported sleeping less than 7 hours or more than 8 hours were considered as experiencing unhealthy sleep. ${ }^{3,18}$ ROC curve analyses were performed to assess the comparative sensitivity and specificity of unhealthy sleep duration (a combination of short and long sleep) versus separating short, average, and long sleep. To perform the ROC analysis sleep duration was categorized in three, short ( $<7 \mathrm{hrs}$.), average ( $7-8 \mathrm{hrs}$.) and long ( $>8 \mathrm{hrs}$ ) sleep durations in the current sample. Based on this finding, we opted to use unhealthy sleep instead of categorizing sleep duration into three distinct sleep durations in our final statistical model.

\section{Covariates}

Information on sociodemographic factors (age, sex, education, income, and obesity), behavioral risk factors (smoking 
and alcohol use), and medical comorbidities (coronary heart disease, diabetes, and stroke) were obtained from the NHIS 2009 dataset. Participants were asked whether they were diagnosed with the above-mentioned substance-related or medical conditions at any point in their lifetime.

\section{Data analysis}

Frequency and measures of central tendency were used to describe the sample. Pearson and Spearman correlations were used to investigate associations between variables of interest. Only factors with a $P$-value $<0.05$ were considered in subsequent multivariate logistic regression analyses, which assessed the association between unhealthy sleep (independent variable) and emotional distress (dependent variable). In all statistical models, we adjusted for effects of sociodemographic factors, behavioral risk factors, and medical comorbidities. All analyses were conducted using IBM SPSS 20.0 (IBM Corporation, Armonk, NY, USA), applying recommended sampling weights provided by NHIS to account for the use of complex sampling strategies.

\section{Results}

Of the sample $(\mathrm{n}=27,731), 51.7 \%$ of the participants were female; $83.1 \%$ were white and $16.9 \%$ were black. Descriptive analyses showed that the majority of the sample (76.5\%) was between the ages of 20 and 60 years; approximately half were married with a spouse in the household and $21.4 \%$ were never married; $85.4 \%$ had at least an eighth grade education; and $31.1 \%$ reported an annual family income below USD $\$ 35,000$. Participants also reported a wide range of behavioral and medical risk factors. Approximately, 42.4\% of the participants reported a history of smoking, $63.3 \%$ reported at least 12 drinks of any type of alcoholic beverage in their entire life, and $29.9 \%$ were obese (body mass index $\geq 30 \mathrm{~kg} / \mathrm{m}^{2}$ ). Few reported chronic health conditions such as history of coronary heart disease (4.6\%), heart problems $(7.8 \%)$, cancer $(8.1 \%)$, diabetes $(9.0 \%)$, and arthritis $(22.9 \%)$, but $28.9 \%$ reported a history of hypertension (28.9\%). With regards to the main variables of interest, $11 \%$ of the participants reported emotional distress and $37.6 \%$ reported unhealthy sleep (Table 1).

Unadjusted logistic regression analysis showed that individuals with emotional distress had $76 \%$ greater odds of reporting unhealthy sleep (odds ratio $[\mathrm{OR}]=1.76,95 \%$ confidence interval $[\mathrm{CI}]=1.63,1.91, P<0.001)$. When effects of sociodemographic variables (ie, age, sex, race/ethnicity, marital status, family income, and education) and healthrisk behavior (ie, smoking history and alcohol use over the past 12 months) were adjusted in the regression model, the odds were reduced to $66 \%(\mathrm{OR}=1.66,95 \% \mathrm{CI}=1.53,1.80$, $P<0.001$ ) (Table 2). When effects of both sociodemographic and medical factors (ie, body mass index, history of alcohol consumption, smoking status, and chronic diseases including arthritis, hypertension, diabetes, cancer, coronary heart disease and heart conditions) were adjusted, the odds of an individual with emotional distress reporting unhealthy sleep were further reduced to $55 \%(\mathrm{OR}=1.55,95 \% \mathrm{CI}=1.42,1.68$, $P<0.001$ ) (Table 3).

\section{Discussion}

Findings from our study, using a nationally representative sample of US adult Americans, are important for two reasons. First, individuals with emotional distress are more likely to report unhealthy sleep in a 24-hour cycle. Secondly, individuals with emotional distress were 1.5 times more likely to report unhealthy sleep duration $(<7$ or $>8$ hours). Of note, this association was independent of known sociodemographic, behavioral, and medical risk factors.

Table I Characteristics of adult participants $(\mathrm{N}=27,73$ I) from the NHIS 2009 dataset

\begin{tabular}{|c|c|}
\hline Variables & (\%) \\
\hline Emotional distress & 11.0 \\
\hline Unhealthy sleep & 37.6 \\
\hline \multicolumn{2}{|l|}{ Age (years) } \\
\hline $20-40$ & 38.0 \\
\hline $4 I-60$ & 38.5 \\
\hline $61-80+$ & 23.5 \\
\hline \multicolumn{2}{|l|}{ Sex } \\
\hline Male & 48.3 \\
\hline Female & 51.7 \\
\hline \multicolumn{2}{|l|}{ Race } \\
\hline White & 83.1 \\
\hline Black & 16.9 \\
\hline \multicolumn{2}{|l|}{ Marital status } \\
\hline Married spouse in household & 53.3 \\
\hline Never married & 21.4 \\
\hline Smoking status & 42.4 \\
\hline Reported at least 12 alcoholic drinks in entire life & 63.3 \\
\hline Income $(<$ USD $\$ 35,000)$ & 31.1 \\
\hline Family education (at least eighth grade) & 85.4 \\
\hline BMI (reference: obese) & 29.9 \\
\hline Hypertension & 28.9 \\
\hline Coronary heart disease & 4.60 \\
\hline Heart problems & 7.80 \\
\hline Cancer & 8.10 \\
\hline Diabetes & 9.00 \\
\hline Arthritis & 22.9 \\
\hline
\end{tabular}

Notes: Emotional distress: $\geq 13$ on the K6 scale; unhealthy sleep: $<7$ or $>8$ hours of sleep per day; race: white only, black only; smoking status: $100+$ cigarettes in lifetime; BMI: obese $\geq 30 \mathrm{~kg} / \mathrm{m}^{2}$; hypertension: ever had a hypertension diagnosis; coronary heart disease: ever had; heart problems: ever had; cancer: ever had; diabetes: ever had type 2 diabetes; arthritis: ever had.

Abbreviations: BMI, body mass index; K6, Kessler 6 Mental Health Scale; NHIS, National Health Interview Survey. 
Table 2 Multivariate-adjusted logistic regression analysis indicating ORs for emotional distress associated with unhealthy sleep in the NHIS 2009 dataset $(\mathrm{N}=27,73 \mathrm{I})$

\begin{tabular}{|c|c|c|c|c|}
\hline \multirow[t]{3}{*}{ Variables } & \multicolumn{3}{|c|}{ Unhealthy sleep (sleep duration $<7$ or $>8$ hours) } & \multirow[t]{3}{*}{$P$-value } \\
\hline & \multirow[t]{2}{*}{ OR } & \multicolumn{2}{|l|}{$95 \% \mathrm{Cl}$} & \\
\hline & & Lower & Upper & \\
\hline Emotional distress & 1.661 & 1.530 & 1.803 & $<0.001$ \\
\hline Age & 1.047 & 1.030 & 1.063 & $<0.001$ \\
\hline Sex (reference: male) & 1.010 & 0.956 & 1.067 & 0.72 \\
\hline Race & 1.504 & 1.403 & 1.611 & $<0.001$ \\
\hline Marital status & 1.029 & 1.018 & 1.039 & $<0.001$ \\
\hline $\begin{array}{l}\text { Smoking status } \\
\text { (reference: never) }\end{array}$ & 0.856 & 0.836 & 0.877 & $<0.001$ \\
\hline $\begin{array}{l}\text { Alcohol in } 12 \text { months } \\
\text { (reference: never) }\end{array}$ & 1.066 & 1.016 & 1.119 & 0.010 \\
\hline Income (reference: < USD $\$ 35,000$ ) & 0.999 & 0.998 & 1.000 & 0.187 \\
\hline $\begin{array}{l}\text { Family education (reference: } \\
\text { high school) }\end{array}$ & 0.990 & 0.984 & 0.997 & 0.004 \\
\hline
\end{tabular}

Notes: Unhealthy sleep: $<7$ or $>8$ hours of sleep per day; emotional distress: $\geq 13$ on the K6 scale; age: $20-40,41-60$, and $61-80+$ years; race: white as reference, black only; marital status: married spouse in the household, divorced, widowed, separated, never married, living with partner; smoking status: ever smoked I00+ cigarettes in lifetime; alcohol: ever had at least 12 drinks of any type of alcoholic beverage; education: less than or equal to eighth grade, ninth-12th grade, GED, High School Diploma, some college AA degree, BA degree, Masters, professional or doctoral degree. Adjusted factors included age, sex, race/ethnicity, and smoking marital status, alcohol use, income, and family education.

Abbreviations: $\mathrm{Cl}$, confidence interval; GED, general educational development; K6, Kessler 6 Mental Health Scale; NHIS, National Health Interview Survey; OR, odds ratio.

These findings are consistent with evidence suggesting that emotional distress influences sleep duration. ${ }^{15,22}$ Furthermore, use of emotional distress, rather than a general psychosocial stress construct, in our study adds a unique insight on the possible debilitating effects of emotional distress on sleep duration.
Although our study did not investigate the mechanisms underlying association of emotional distress with unhealthy sleep duration, it adds to the literature on neurobiological and psychological sequelae of unhealthy sleep (short or long sleep duration). It has been shown that short and long sleep durations negatively affect neurobiological and

Table 3 Multivariate-adjusted logistic regression analysis indicating ORs for emotional distress associated with unhealthy sleep in the NHIS 2009 dataset $(\mathrm{N}=27,73 \mathrm{I})$

\begin{tabular}{|c|c|c|c|c|}
\hline \multirow[t]{3}{*}{ Variables } & \multicolumn{3}{|c|}{ Unhealthy sleep (sleep duration $<7$ or $>8$ hours) } & \multirow[t]{3}{*}{$P$-value } \\
\hline & \multirow[t]{2}{*}{ OR } & \multicolumn{2}{|l|}{$95 \% \mathrm{Cl}$} & \\
\hline & & Lower & Upper & \\
\hline Emotional distress & 1.548 & 1.423 & 1.684 & $<0.001$ \\
\hline Age & 0.982 & 0.964 & 1.001 & 0.070 \\
\hline Sex (reference: male) & 0.991 & 0.937 & 1.048 & 0.743 \\
\hline Race & 1.470 & 1.369 & 1.578 & $<0.001$ \\
\hline Marital status & 1.030 & 1.019 & $\mathrm{I} .04 \mathrm{I}$ & $<0.001$ \\
\hline Smoking status (reference: never) & 0.863 & 0.842 & 0.884 & $<0.001$ \\
\hline Alcohol in 12 months (reference: never) & 1.052 & 1.001 & 1.106 & 0.045 \\
\hline Annual income (reference: $<$ USD $\$ 35,000$ ) & 1.000 & 0.998 & 1.001 & 0.481 \\
\hline Family education (reference: high school) & 0.992 & 0.985 & 0.998 & 0.016 \\
\hline BMI (reference: obese) & 1.201 & 1.132 & 1.275 & $<0.001$ \\
\hline Hypertension & 1.077 & 1.001 & $1.15 \mathrm{I}$ & 0.028 \\
\hline Coronary heart disease (reference: none) & 1.112 & 0.979 & 1.264 & 0.103 \\
\hline Heart problems (reference: none) & 1.169 & 1.057 & 1.293 & 0.002 \\
\hline Cancer (reference: none) & 1.178 & 1.069 & 1.298 & 0.001 \\
\hline Diabetes (reference: none) & 1.093 & 0.996 & 1.199 & 0.060 \\
\hline Arthritis (reference: none) & 1.431 & 1.337 & 1.532 & $<0.001$ \\
\hline
\end{tabular}

Notes: Unhealthy sleep: $<7$ or $>8$ hours of sleep per day; emotional distress: $\geq 13$ on the K6 scale; age: $20-40,41-60$, and $61-80+$ years; race: white as reference, black only; smoking: ever smoked $100+$ cigarettes in lifetime; Marital status, married is reference; BMl: obese $\geq 30 \mathrm{~kg} / \mathrm{m}^{2}$; hypertension: ever had a hypertension diagnosis by a physician; coronary heart disease: ever had a coronary heart disease; heart problems: ever had; cancer: ever had; diabetes: ever had a type 2 diabetes diagnosis in lifetime; arthritis: ever had. Adjusted variables include: age, sex, race, marital status, smoking status, alcohol, annual income, family education, BMI, Hypertension, Coronary Heart disease, Heart problems, Cancer, Diabetes and arthritis.

Abbreviations: BMI, body mass index; Cl, confidence interval; K6, Kessler 6 Mental Health Scale; NHIS, National Health Interview Survey; OR, odds ratio. 
psychophysiological reactivity and functional alteration in the amygdala, hypothalamus-pituitary-adrenocortical axis, and prefrontal cortex, which in turn may cause unhealthy sleep. ${ }^{25,26}$ Our findings are also consistent with three relevant models that explain adverse effects of short sleep (Harvey's presleep cognitive activity, ${ }^{27}$ Riemann's hyperarousal model of insomnia, ${ }^{28}$ and Fernandez-Mendoza's cognitive-emotional hyperarousal $\operatorname{model}^{29}$ ), which provide mechanistic insights as to how and why emotional distress might be related to sleep duration. Harvey's model suggests that the short sleep duration experienced by insomniacs is induced by presleep worry about their day and daytime worry about not getting enough sleep. According to Riemann et al, insomnia - difficulty falling asleep, maintaining sleep, and early-morning awakenings $^{28}$ - is likely due to hyperarousal processes induced/ mediated by emotional distress during the day and night. Fernandez-Mendoza et al suggested that the hyperarousal nature of insomnia symptoms are due to inherited cognitive and emotional factors. Particularly, parents who reported high levels of perceived stress, depression, anxiety, and presleep cognitive and somatic arousal were three to seven times more likely to have children vulnerable to stress-based insomnia. ${ }^{29}$ With regard to long sleep duration, Riemann et $\mathrm{al}^{30}$ found that depressed individuals had reduced slow wave sleep, shortened rapid eye movement (REM) latency, a longer first REM period, and increased REM density. Several theoretical models, such as the two-process model of sleep and sleep regulation, the glomerular filtration rate/chronic renal failure imbalance model, and the reciprocal model of non-REM and REM sleep regulation explain how emotional distress impacts long sleep. ${ }^{30}$

We identified several limitations to our findings. First, since the NHIS uses self-reported data, the findings might be susceptible to reporting bias. However, we should note that polysomnography or actigraphy, which would provide objective sleep assessment, is prohibitively costly and limits the ability to obtain nationally representative sleep data, as achieved by the NHIS. Second, the use of cross-sectional design does not permit the establishment of cause-and-effect relationships between emotional distress and unhealthy sleep. Third, we were not able to adjust for the covarying effects that occupational and work shift status may have on sleep duration. Fourth, omission of substance in our analysis highlights another limitation, as emotional distress, substance use, and sleep disturbances are likely associated with each other and are comorbid conditions. ${ }^{31}$ Substance use could act as a mask for distressing symptoms of anxiety and depression, in which case the emotional distress of this population would not have been accounted for given the limitation of the instrument. Despite these limitations, our findings offer strong evidence supporting independent relationships between emotional distress and unhealthy sleep durations. Future studies should investigate the longitudinal effects of psychological well-being on unhealthy sleep, since recent evidence utilizing cross-lagged autoregressive longitudinal analysis suggests that self-reported psychological well-being predicts unhealthy sleep duration.

\section{Conclusion}

Individuals with emotional distress show a 50\% greater likelihood of experiencing unhealthy sleep (sleep duration $<7$ or $>8$ hours). Independent of the effects of low socioeconomic status, high body mass index, history of alcohol consumption, smoking behavior, arthritis, hypertension, diabetes, cancer, coronary heart disease and heart condition, emotional distress was independently associated with unhealthy sleep. Thus, the assessment of emotional distress should be performed among individuals experiencing unhealthy sleep durations. Future studies should investigate: 1) the relationship between the severity and duration of emotional distress and sleep duration; 2) whether sleep duration moderates or mediates the contribution of genetic influence on emotional distress; 3 ) whether emotional distress moderates or mediates the contribution of genetic influence on sleep duration; and 4) the possibility of exploring the interrelationship of substance-related behaviors, emotional distress, and sleep disturbance.

\section{Acknowledgment}

This work was supported by funding from the NIH (R01MD007716, U54NS081765, and K24HL111315).

\section{Disclosure}

The authors report no financial conflict or conflicts of interest in this work.

\section{References}

1. Liu Y, Wheaton AG, Chapmna DP, Croft JB. Sleep duration and chronic diseases among US adults age 45 years and older: evidence from the 2010 Behavioral Risk Factor Surveillance System. Sleep. 2013; 36(10):1421-1427.

2. Cappuccio FP, Cooper D, D'Elia L, Strazzullo P, Miller MA. Sleep duration predicts cardiovascular outcomes: a systematic review and metaanalysis of prospective studies. Eur Heart J. 2011;32(12):1484-1492.

3. Buxton OM, Marcelli E. Short and long sleep are positively associated with obesity, diabetes, hypertension, and cardiovascular disease among adults in the United States. Soc Sci Med. 2010;71(5):1027-1036.

4. Papp KK, Penrod CE, Strohl KP. Knowledge and attitudes of primary care physicians toward sleep and sleep disorders. Sleep Breath. 2002;6(3):103-109. 
5. Grandner MA, Martin JL, Patel NP, et al. Age and sleep disturbances among American men and women: data from the U.S. Behavioral Risk Factor Surveillance System. Sleep. 2012;35(3):395-406.

6. Stamatakis KA, Kaplan GA, Roberts RE. Short sleep duration across income, education and race/ethnicity groups: population prevalence and growing disparities over 34 years of follow-up. Ann Epidemiol. 2007; 17(12):948-955.

7. Kang HH, Kang JY, Ha JH, et al. The associations between anthropometric indices and obstructive sleep apnea in a Korean population. PLoS One. 2014;9(12):e114463. doi:10.1371/journal.pone.0114463.

8. Young T, Peppad PE, Taheri S. Excess weight and sleep-disordered breathing. J Appl Physiol. 2005;99(4):1592-1599.

9. Schoenborn CA, Adams PF. Sleep duration as a correlate of smoking, alcohol use, leisure-time physical inactivity and obesity among adults: United States, 2004-2006. NCHS Health E-Stats; 2008. Available from: http://www.cdc.gov/nchs/products/pubs/pubd/hestats/sleep04-06/ sleep04-06.htm. Accessed on August 14, 2014.

10. Kim EJ, Dimsdale JE. The effect of psychosocial stress on sleep: a review of polysomnographic evidence. Behav Sleep Med. 2007;5(4): 256-278.

11. te Velde SJ, van der Aa N, Boomsma DI, et al. Genetic and environmental influences on individual differences in sleep duration during adolescence. Twin Res Hum Genet. 2013;16(6):1015-1025.

12. Basta M, Chrousos GP, Vela-Bueno A, Vgontzas AN. Chronic insomnia and stress system. Sleep Med Clin. 2007;2(2):279-291.

13. Weinstock TG, Rosen CL, Marcus CL, et al. Predictors of obstructive sleep apnea severity in adenotonsillectomy candidates. Sleep. 2014; 37(2):261-269.

14. Zhang L, Samet J, Caffo B, Punjabi NM. Cigarette smoking and nocturnal sleep architecture. Am J Epidemiol. 2006;164(6):529-537.

15. Roberts RE, Duong HT. The prospective association between sleep deprivation and depression among adolescents. Sleep. 2014;37(2): 239-244.

16. Walker MP. The role of sleep in cognition and emotion. Ann N Y Acad Sci. 2009;1156:168-197.

17. Goncharenko AM. Electrophysiological investigation of sleep in shift workers exposed to emotional stress due to work. Hum Physiol. 1979;5(4):468-474.

18. Krueger PM, Friedman EM. Sleep duration in the United States: a cross-sectional population-based study. Am J Epidemiol. 2009;169(9): 1052-1063.
19. Kaneita Y, Ohida T, Osaki Y, et al. Association between mental health status and sleep status among adolescents in Japan: a nationwide crosssectional survey. J Clin Psychiatry. 2007;68(9):1426-1435.

20. Bao AM, Meynen G, Swaab DF. The stress system in depression and neurodegeneration: focus on the human hypothalamus. Brain Res Rev. 2008;57(2):531-553.

21. Sarchiapone M, Mandelli L, Carli V, et al. Hours of sleep in adolescents and its association with anxiety, emotional concerns, and suicidal ideation. Sleep Med. 2014;15(2):248-254.

22. Blasco-Fontecilla H, Alegria AA, Lopez-Castroman J, et al. Short selfreported sleep duration and suicidal behavior: a cross-sectional study. $J$ Affect Disord. 2011;133(1-2):239-246.

23. Kessler RC, Andrews G, Colpe LJ, et al. Short screening scales to monitor population prevalence and trend in non-specific psychological distress. Psychol Med. 2002;32(6):959-976.

24. Feketich AK, Binkley PF. Psychological distress and cardiovascular disease: results from the 2002 National Health Interview Survey. Eur Heart J. 2005;26(18):1923-1929.

25. Kalmbach DA, Pillai V, Roth T, Drake CL. The interplay between daily affect and sleep: a 2-week study of young women J Sleep Res. 2014; 23(6):636-645.

26. Prather AA, Bogdan R, Hariri AR. Impact of sleep quality on amygdala reactivity, negative affect, and perceived stress. Psychosom Med. 2013; 75(4):350-358.

27. Harvey AG. Pre-sleep cognitive activity: a comparison of sleeponset insomniacs and good sleepers. Br J Clin Psychol. 2000;39(3): 275-286.

28. Reimann D, Spiegelhalder K, Feige B, et al. The hyperarousal model of insomnia: a review of the concept and its evidence. Sleep Med Rev. 2010;14(1):19-31.

29. Fernandez-Mendoza J, Shaffer ML, Olavarrieta-Bernardino S, et al. Cognitive-emotional hyperarousal in the offspring of parents vulnerable to insomnia: a nuclear family study. J Sleep Res. 2014;23(5):489-498.

30. Riemann D, Berger M, Voderholer U. Sleep and depression - results from psychobiological studies: an overview. Biol Psychol. 2001; 57(1-3):67-103.

31. Foster JH, Peters TJ, Kind P. Quality of life, sleep, mood and alcohol consumption: a complex interaction. Addict Biol. 2002;7(1):55-65.
Neuropsychiatric Disease and Treatment

\section{Publish your work in this journal}

Neuropsychiatric Disease and Treatment is an international, peerreviewed journal of clinical therapeutics and pharmacology focusing on concise rapid reporting of clinical or pre-clinical studies on a range of neuropsychiatric and neurological disorders. This journal is indexed on PubMed Central, the 'PsycINFO' database and CAS,

\section{Dovepress}

and is the official journal of The International Neuropsychiatric Association (INA). The manuscript management system is completely online and includes a very quick and fair peer-review system, which is all easy to use. Visit http://www.dovepress.com/testimonials.php to read real quotes from published authors. 